\title{
CHRONIC MYELOID LEUKEMIA IN PATIENT WITH THE KLINEFELTER SYNDROME
}

\author{
S.V. Andreieva ${ }^{1, *}$, K.V. Korets ${ }^{2}$, O.A. Kyselova ${ }^{1}$, O.E. Ruzhinska ${ }^{2}$, I.M. Serbin ${ }^{3}$ \\ ${ }^{1}$ State Institution «Institute of Haematology and Transfusiology of NAMS of Ukraine», Kyiv 04060, Ukraine \\ ${ }^{2}$ Government Institution «The Scientific-Practical Children's Cardiac Centre the Ministry of Health Care \\ of Ukraine» (UCCC), Kyiv 04050, Ukraine \\ ${ }^{3}$ City Hospital No 9, Kyiv 04060, Ukraine
}

Aim: Genetic inborn along with acquired diseases arise due to the lesions in genome of multipotent hematopoietic stem cells. The aim was to study an influence of constitutional anomaly, Klinefelter syndrome, and additional structural rearrangements on the BCR-ABL tyrosine kinase inhibitor targeted therapy efficacy. Material and Methods: We describe a 32-year-old male patient with chronic myeloid leukemia (CML) who was detected to have sex chromosomal abnormality during evaluation for Philadelphia chromosome. Results: At diagnosis of CML, two clones were detected by standard cytogenetic investigation of bone marrow cells: 1) clone with translocation $t(9 ; 22)(q 34 ; q 11)$, with two sex $X$ chromosomes and absence sex chromosome $Y ; 2)$ clone with $t(9 ; 22)$ and unbalanced $t(Y ; 20)(q 11 ; q 13)$. Analysis of blast transformed lymphocytes from peripheral blood showed karyotype 47,XXY. Monitoring of targeted therapy with second generation inhibitor of BCR-ABL tyrosine kinase indicated a cytogenetic remission and absence of BCR-ABL1 fusion signals after 11 months. Conclusions: Absence of translocation $\mathrm{t}(9 ; 22)(\mathrm{q} 34 ; \mathrm{q} 11)$ in blast transformed T-lymphocytes at diagnosis of CML evidences that this translocation may appear not only at the level of multipotent haemopoietic cell progenitors but also may have oligo lineage myeloid origin. Presence of additional structural chromosomal abnormality in the clone with $t(9 ; 22)(q 34 ; q 11)$ does not affect the efficacy of therapy with the use of second generation BCR-ABL tyrosine kinase inhibitor.

Key Words: Klinefelter syndrome, chronic myeloid leukemia, additional chromosomal abnormalities, targeted therapy.

Chronic myeloid leukemia $(\mathrm{CML})$ is an acquired disease that arises due to the lesions in genome of multipotent hematopoietic stem cell, and makes up approximately $14 \%$ of all types of leukemias. CML is a disease of middle age with manifestation peak in 40-50 years old. The cause of the disease is not currently established, but in the history of some patients the chemotherapy and radiation have been registered [1, 2]. CML cytogenetic feature is the translocation $\mathrm{t}(9 ; 22)(\mathrm{q} 34 ; \mathrm{q} 11)$ with the breakpoints in chromosome 9 in the band q34 (ABL gene) and chromosome 22 in the band q11 (BCR gene). The latter was designated the Philadelphia chromosome ( $\mathrm{Ph}^{\prime}$-chromosome) [3]. As a result of this translocation, two chimeric genes are formed: $A B L 1-B C R$ on chromosome 9 and $B C R-A B L 1$ on chromosome 22 . Considering that the anomaly occurs at an early precursor of hematopoietic and lymphoid tissues, it is believed that this rearrangement might be registered in all bone marrow (BM) cells, including the peripheral blood (PB) T-lymphocytes stimulated by phytohemagglutinin (PHA) [4]. Other authors indicate lack of translocation $\mathrm{t}(9 ; 22)(\mathrm{q} 34 ; \mathrm{q} 11)$ in T-lymphocytes [5].

The use of inhibitors of BCR-ABL tyrosine kinase allows achieving both the clinical and hematologic, and also long-term cytogenetic and molecular genetic remission. This also concerns to the cases of variant translocations $t(9 ; 22)$ and additional abnormalities in the clone with $\mathrm{t}(9 ; 22)$ in BM cells at diagnosis of $\mathrm{CML}[2,6]$.

Submitted: July 14, 2016.

*Correspondence: Fax: +38 (044) 4402722

E-mail: office@immd.kiev.ua

Abbreviations used: $\mathrm{BM}$ - bone marrow; $\mathrm{CML}$ - chronic myeloid leukemia; KS - Klinefelter syndrome; PB - peripheral blood; $\mathrm{PHA}$ - phytohemagglutinin.
To confirm the genetic remission following the therapy with inhibitors of BCR-ABL tyrosine kinase, cytogenetic and molecular genetic control is always required. Achievement of the cytogenetic remission in 3 months of treatment with first generation BCR-ABL tyrosine kinase inhibitors as well as molecular genetic remission (one abnormal cell in $10^{5}$ of BM cells) during the twelve months therapy, are considered as the gold standard of effective CML therapy [7].

Klinefelter syndrome (KS) is the mostabundantanomaly of sex chromosomes in men occuring in 1:500 cases [8]. The classic form of KS is described as 47, XXY. However, sometimes the increase in sex chromosomeXnumber up to four copies and sex chromosome $Y$ up to two copies, or mosaic form $47, \mathrm{XXY} / 46, \mathrm{XY}$ appearance are reported [9]. The cases of CML registered in patients with KS are quite rare [10-14]. Among these cases, two patients are described in whom the effectiveness of BCR-ABL tyrosine kinase inhibitors-based therapy was assessed. In one of these cases, the patient remained in complete hematological and major molecular remission after 2 years of the therapy with first generation tyrosine kinase inhibitor [13], while in the other case no response to the same inhibitor was achieved and persistent molecular genetic alterations were documented during the therapy with the II generation inhibitor [15].

We, therefore, aimed to study an influence of the constitutional anomaly, KS, and additional structural rearrangements on the $B C R-A B L$ tyrosine kinase inhibitor targeted therapy efficacy.

\section{MATERIALS AND METHODS}

The patient $\boldsymbol{D}$. , 32-year-old male, was referred to hematologist for the first time in October 2013 complaining 
of the expressed weakness, skin pallor and weight loss during the year (height $175 \mathrm{~cm}$, weight $104 \mathrm{~kg}$ ). The patient gave written informed consent for the publication of his data analyses. Primary observation showed that the patient had narrow shoulders and wide hip. The PB contained $126.5 \cdot 10^{9} / \mathrm{lleukocytes}$ and $39.0 \mathrm{~g} / \mathrm{l} \mathrm{hemo-}$ globin. Spleen size measured by ultrasound investigation of peritoneum was $60 \times 120 \mathrm{~mm}$. To diagnose the patient, the sternum puncture has been done along with the BM morphological evaluation and further cytogenetic investigation for confirmation of the probable CML diagnosis. BM and PB cell counts are presented in the Table.

Table. BM and PB cells indexes

\begin{tabular}{ll}
\hline \multicolumn{1}{c}{ BM } & \multicolumn{1}{c}{ PB } \\
\hline Blast cells, 6.0\% & Myelocytes, 23\% \\
Leukocyte-erythrocyte ratio 21:1 & Metamyelocytes, 20\% \\
Neutrophil maturity index 1.0 & Immature granulocytes, 15\% \\
& Neutrophilic granulocytes, 34\% \\
& Lymphocytes, 8\% \\
\hline
\end{tabular}

For cytogenetic study of BM cells, the slides of metaphase chromosomes were prepared according to the conventional method followed by GTG staining. Considering that besides classic translocation $\mathrm{t}(9 ; 22)$ ( $\mathrm{q} 34 ; \mathrm{q} 11$ ) typical for CML, mosaics of sex chromosomes was detected in patient, both the molecular genetic assay (FISH) on BM cells and karyotyping of PB blast transformed cells were performed. To define the nature of additional sex chromosome $\mathrm{X}$, the conventional cytogenetics of blast transformed lymphocytes was carried out after $72 \mathrm{~h}$ cultivation of PB and addition of PHA in the medium. Slides were stained by GTG-method. Presence of clonal chromosomal abnormalities was described according to ISCN 2013, when two or more metaphase plates had identical structural or additional (trisomy) chromosome abnormalities and also in appearance of three or more metaphase plates with identical chromosomes monosomies [16]. The presence of translocation $t(9 ; 22)(q 34 ; q 11)$ during the targeted therapy was revealed by conventional cytogenetics and after fluorescence staining per se (on slide) in interphase nuclei (i-FISH). i-FISH assay was carried out on interphase cell nuclei after $24 \mathrm{~h}$ cultivation. The probe set Vysis LSI BCR/ABL Dual Color, Dual Fusion Translocation Probe (Abbott, USA) was used.

\section{RESULTS AND DISCUSSION}

Standard cytogenetic study on BM suspension cells revealed mosaic karyotype consisting of three clones: 46,XX,-Y,t(9;22)(q34;q11) [15]/ 46,XX,t(Y;20) $(q 11 ; q 13), t(9 ; 22)(q 34 ; q 11),-\operatorname{der}(Y) t(Y ; 20)(q 11 ; q 13)$ [3] $/ 4 n \pm$ [2]. The first clone with two sex $X$ chromosomes, absence of sex $Y$ chromosome and translocation $t(9 ; 22)$ (q34; q11) was detected in 15 metaphase plates (Fig. 1). The second clone with two sex X chromosomes, unbalanced structural rearrangement, namely translocation between chromosomes $Y$ and 20 and loss of derivative chromosome due to translocation between chromosomes Yand 20 was detected in three metaphase plates (Fig. 2), and the third clone (near tetraploid) was detected in two metaphase plates. The results allowed us to suspect that the patient might have innate genetic disease, the KS. Moreover, it is known that during formation of abnor- mal karyotypes the losses of sex chromosomes could occur. Therefore, cytogenetic study on blast transformed PB lymphocytes was carried out to confirm the constitutional nature of additional sex chromosome $X$. The study showed karyotype $47, X X Y$ in 20 analyzed metaphase plates (Fig. 3). That was confirmed by cytogenetics as KS. The results suggest the involvement of sex chromosome $Y$ in the formation of two abnormal clones with translocation $\mathrm{t}(9 ; 22)$. In the first clone with a translocation $t(9 ; 22)$, a loss of sex chromosome Ywas observed; in the second clone with a translocation $\mathrm{t}(9 ; 22)$, the unbalanced translocation was registered involving sex chromosome Yand chromosome 20 with the loss of derivative chromosomeY, which was formed as a result of this translocation. The episode of the formation of different clones in BM cells karyotype at CML diagnosis in patient with the KS is reported for the first time. The absence of $t(9 ; 22)$ in PHA blast transformed PB T-lymphocytes at diagnosis of CML has questioned the previous statement that this translocation can be detected in lymphocytes. Perhaps in some cases the translocation could occur not only at the level of multipotent hematopoietic progenitor cell but also may have oligo lineage myeloid origin.

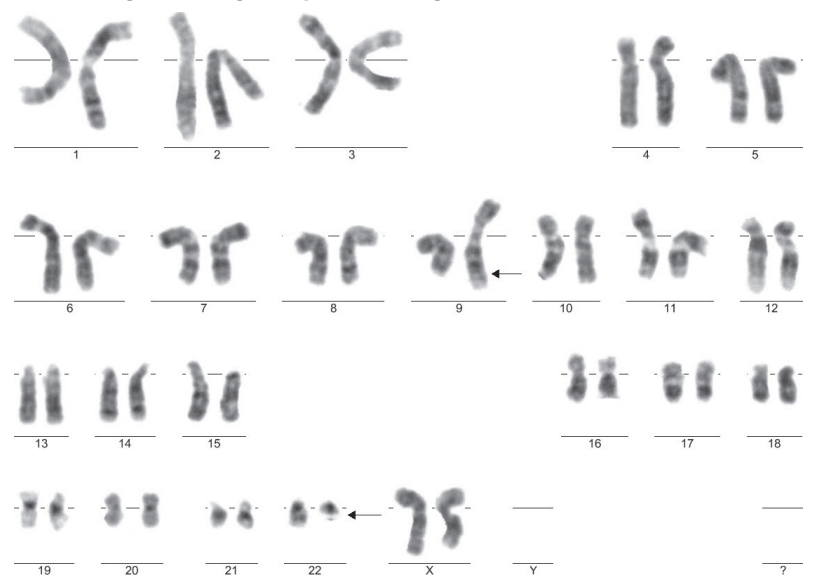

Fig. 1. Cytogenetic analysis of BM cells shows a clone with two sex chromosomes Xand balanced translocation $\mathrm{t}(9 ; 22)$ (q34;q11)

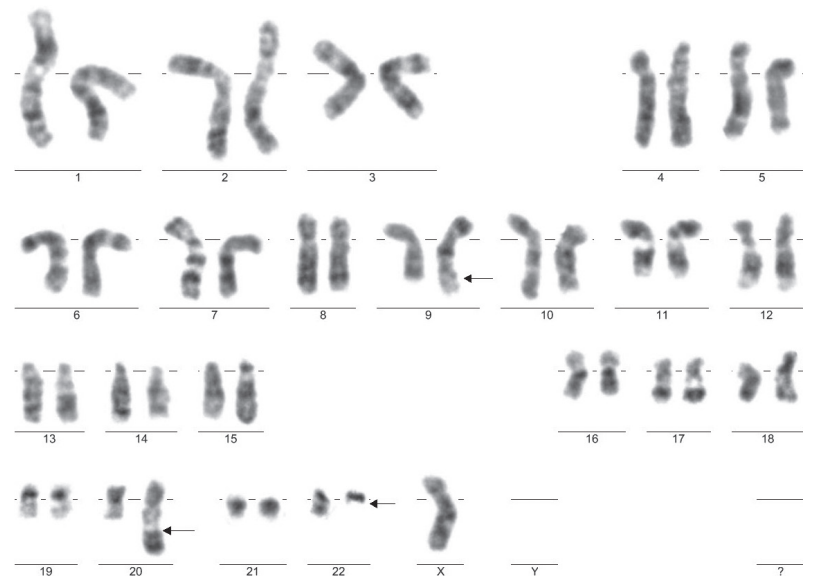

Fig. 2. Cytogenetic analysis of BM cells shows a clone with one sex chromosome $X$, balanced translocation $\mathrm{t}(9 ; 22)(\mathrm{q} 34 ; \mathrm{q} 11)$ and unbalanced translocation $\mathrm{t}(\mathrm{Y} ; 20)(\mathrm{q} 11 ; \mathrm{q} 13)$

Since November 2013, the patient was scheduled to hydroxyurea chemotherapyata dose of $4000 \mathrm{mg} /$ day; there was a reduction in the leucocyte count to normal value while anemia remained. 


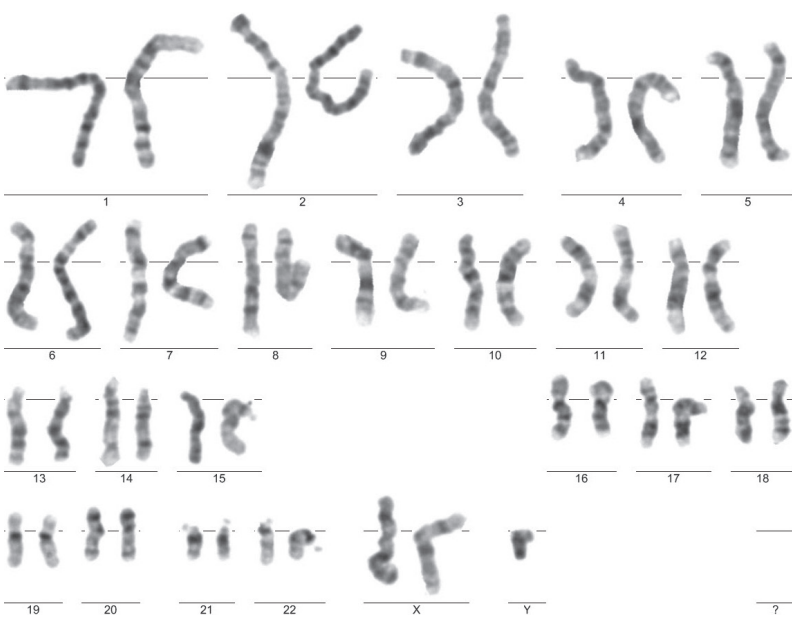

Fig. 3. Conventional cytogenetics shows karyotype $47, X X Y$ in blast transformed T-lymphocytes of PB

Since December 2014, the therapy with the II generation inhibitor of BCR-ABL tyrosine kinase at a dose of $600 \mathrm{mg} /$ day has been prescribed, which is still continued. As a result of the treatment, the patient recovered to normal PB and myelogram parameters, spleen size reduced to $100 / 60 \mathrm{~mm}$ on ultrasound examination, and significant increase in weight $(125 \mathrm{~kg})$ was observed. Cytogenetic and molecular cytogenetic monitoring of the therapy effectiveness was conducted in 11 months. Analysis of metaphase chromosomes in BM cells has revealed the karyotype containing additional sex chromosome X: 47,XXY (in 20 metaphase plates). Simultaneously, iFISH assay was carried out on 200 interphase $\mathrm{BM}$ nuclei, and registered an absence of fusion signals of BCR-ABL1 and ABL1-BCR formed due to $t(9 ; 22)$ (Fig. 4).

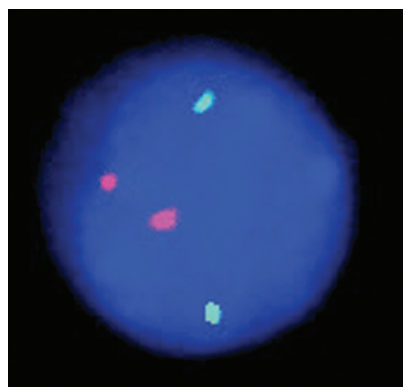

Fig. 4. Molecular cytogenetic study shows normal distribution of $B C R$ and $A B L 1$ signals

Thus, in patient with CML in the presence of constitutional chromosomal abnormality, KS, two independent clones were recorded in BM cells at diagnosis: translocation $\mathrm{t}(9 ; 22)$ in clone with two sex chromosomes $X$ and without sex chromosome $Y$, and translocation $\mathrm{t}(9 ; 22)$ and unbalanced structural rearrangement involving sex chromosome $Y$. The molecular cytogenetic remission was achieved following 11 months of the treatment with second generation inhibitor of BCR-ABL tyrosine kinase. Absence of translocation $\mathrm{t}(9 ; 22)(\mathrm{q} 34 ; \mathrm{q} 11)$ in blast transformed T-lymphocytes may indicate that not all hematopoietic cells carry this abnormality. The data would be important to assess the effectiveness of targeted therapy for patients with the Copyright @ Experimental Oncology, 2016
KS in the presence of additional structural abnormalities in clone with translocation $t(9 ; 22)$.

\section{ACKNOWLEDGEMENTS}

We thank Prof. Danylo Gluzman, RE Kavetsky IEPOR of the NAS of Ukraine, for the confirmation of the diagnosis and disease stage. We are grateful to Dr. Olena Alkhimova for the valuable advice and assistance.

\section{REFERENCES}

1. D’Antonio J. Chronic myelogenous leukemia. Clin J Oncol Nurs 2005; 9: 535-53.

2. Hochhsus A, Lahaye T, Kreil S, et al. Imatinib (STI571) for the treatment of chronic myeloid leukemia (CML). In: Acute leukemia IX. W. Hiddemannet et al., eds. SpringerVerlag Berlin Heidelberg, 2003: 109-16.

3. http://atlasgeneticsoncology.org.

4. Haferlach N, Winkemann M, Nickenig C, et al. Which compartments are involved in Philadelphia-chromosome positive chronic myeloid leukaemia? An answer at the single cell level by combining May-Grünwald-Giemsa staining and fluorescence in situ hybridization techniques. Br J Haematol 1997; 97: 99-106.

5. Bosch GJ, Joosten AM, Kessler JH, et al. Recognition of BCR-ABL positive leukemic blasts by human CD4+ T cells elicited by primary in vitro immunization with a BCR-ABL breakpoint peptide. Blood 1996; 88: 3522-7.

6. Talpaz MO, O'Brein S, Jones D, et al. Molecular responses in patients with chronic myeloid leukemia in chronic phase treated with imatinib mesylate. Clin Cancer Res 2005; 11: 3425-32.

7. European LeukemiaNet (http://www.leukemia-net.org).

8. Toubai T, Tanaka J, Ota S, et al. Allogeneic bone marrow transplantation from an unrelated donor for the treatment of chronic myelogenous leukemia in blast crisis in a patient with Klinefelter's syndrome. Leuk Lymphoma 2004; 45: 829-31.

9. Smyth CM, Bremner WJ. Klinefelter syndrome. Arch Intern Med 1998; 158: 1309-14.

10. Eberl MM, Baer MR, Mahoney MC, et al. Unsuspected Klinefelter syndrome diagnosed during oncologic evaluation: a case series. J Am Board Fam Pract 2005; 18: 132-9.

11. Oguma N, Takemoto M, Oda K, et al. Chronic myelogenous leukemia and Klinefelter's syndrome. Eur J Haematol 1989; 42: 207-8.

12. Adhvaryu SG, Jani KH, Balar DB, Shah PM. Klinefelter syndrome patient with chronic myelogenous leukemia. Cancer Genet Cytogenet 1990; 48: 135-7.

13. Chennuri V, Kashyap R, Tamhankar P, Phadke S. Chronic myeloid leukemia in case of Klinefelter syndrome. Indian J Hum Genet 2014; 20: 69-71.

14. Chakraborty R, Mukkamalla SKR, Singam K, Calderon N. Persistent suboptimal molecular response in a patient with chronic myelogenous leukemia and Klinefelter syndrome. Korean J Intern Med 2014; 29: 827-9.

15. Czepulkowski BH, Bhatt R, Rooney DE. Basic techniques for the preparation and analysis of chromosomes from bone marrow and leukaemic blood. In: DE Rooney, BH Czepulkowski, eds. Human Cytogenetics. Malignancy and acquired abnormalities: a practical approach. Oxford NewYork Tokyo: Oxford University Press, 1992. 25 p.

16. International system for human cytogenetic nomenclature. LG Shaffer, J Mc Gowan-Jordan, M Schmid, eds. Recommendations of the International Standing Committee on Human Cytogenetic Nomenclature. Basel: S Karger, 2013. 140p. 